\title{
A first step to Fluid-Structure interaction inside Solid Propellant Rocket Motors
}

\author{
S. Cerqueira, F. Feyel \& G. Avalon \\ ONERA, Chatillon Cedex, France
}

\begin{abstract}
Self sustained oscillations in Solid Propellant Rocket Motors result from an aeroacoustic phenomenon involving interaction between vortex shedding and acoustics of the chamber. An experimental investigation has been carried out with a cold gas apparatus for acoustic oscillations sustained by vortex shedding. Furthermore, Fluid-Structure interactions have been shown to be of importance when studying pressure oscillations inside Solid Propellant Rocket Motors. Numerical results are compared to preliminary tests undertaken with the use of a non-intrusive measurement technique allowing both displacement and frequency measurements of flexible inhibitors.
\end{abstract}

Keywords: Solid Rocket Propellant Motors, Fluid-Structure, numerical simulation, vortex shedding.

\section{Introduction}

Discovered in the late 1930s, [1], unsteady motions in Solid Propellant Rockets were the first examples discovered of combustion instabilities in propulsion systems. Since that time, extensive work has been done on pressure oscillations motivated, for instance, by low frequency oscillations in the boosters of the European launcher Ariane 5 [2]. Conveniently assembled with segmented grains, large Solid Propellant Rocket Motors (SPRM) have interfaces coated with an inert, slow burning material (named inhibitors). As the propellant burns radially during the firing, the inhibitors protrude into the flow in the form of annular rings giving rise to unstable shear layers.

First suggested by Flandro and Jacobs in 1973, [3], and by Flandro twelve years later in an extension of his previous analysis, [4], periodic vortex shedding may induce pressure oscillations. According to Flandro's work, vortex shedding from 
inhibitors has been identified as a possible mechanism for the excitation of the acoustic modes of a combustion chamber. In one of the earliest papers, Culick and Magiawala [5] carried out studies of flow past inhibitors in a duct. They demonstrated that vortex shedding at a pair of inhibitors may excite acoustic modes by a near coincidence with acoustic mode frequency. Experimental results reported by Dunlap and Brown in the 1980s [6], and later by Brown et al. [7] and Namoto and Culick [8], confirmed the excitation of Acoustic tones under certain flow and geometrical conditions and set the basis of vortex shedding as a significant source of acoustic energy [9].

During the past five decades, many studies on this topic have been conducted either analytically [10], numerically [11] or experimentally with more realistic cold flow apparatus (see [12-14]). In addition, for quantifying the effect of the obstacle deflection on flow, some numerical attempts to simulate inhibitors were conducted in a quasi-steady approach [15], but never been compared to experimental data. More recently, the dynamic response of inhibitors was taken into account in a fully coupled simulation $[16,17]$, but with a speculated constitutive model for inhibitor.

\section{Strategy for Fluid-Structure approach}

Fluid Structure Interaction (FSI) in SPRM is a complex phenomenon involving aeroacoustics interacting with the non-linear mechanical behavior of the inhibitor. The work presented in this paper is part of an overall methodology, which can be divided into three main parts:

- Linear stability theory is used to investigate the stability of the shear layer resulting from the presence of the protruding inhibitors. Based on the understanding gained from this analysis, it will be stated whether vortices can grow due to the instability of the flow and how it could interact with the acoustic field.

- Nonlinear mechanical behavior of the inhibitors is studied in a series of tests in order to quantitatively identify the major features of the stress-strain behavior of such materials. A constitutive model for the observed stressstrain behavior is then developed and compared directly to experimental data.

- Numerical simulations of the FSI are performed, using CEDRE and Zebulon solvers, respectively for fluid and structural subsystems. As a first step, a quasi-static simulation is computed in order to validate the partitioned coupling methodology. This paper mainly deals with this point of the global approach herein described. Once the numerical methodology is validated on the quasi-static case, a fully coupled dynamic simulation will be performed. FSI simulations of the experimental apparatus VALDO will be compared to experimental data and to a phenomenological analytical model. 


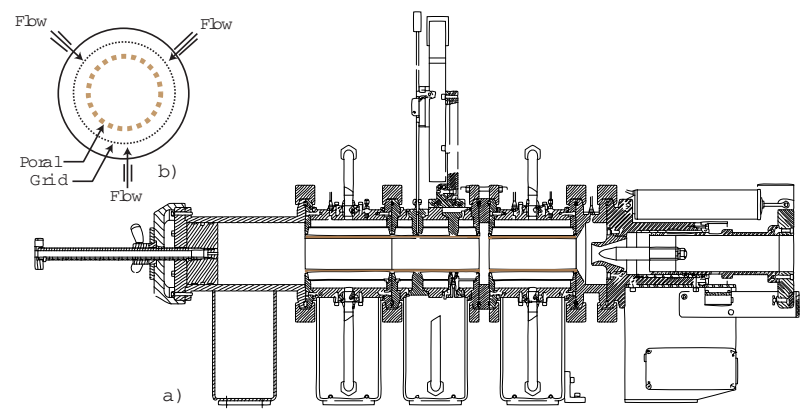

Figure 1: Scheme of the VALDO setup: a) general view, three injecting elements and different cavities mounted; b) sketch of an injecting element.

\section{Experimental investigation}

\subsection{VALDO setup}

The experimental facility VALDO (sketched in Figure 1) is a modular axisymmetric cold flow setup similar to the device imagined by Brown et al. [7]. A detailed description of the setup may be found in [18]. The experimental setup is composed of injecting modules equipped, in their central part, with porous tubes. Air is injected at three different azimuthal angles for each element used and is assumed to be spread out uniformly with respect to the axial direction and the azimuthal one. The uniformity of the mass flow rate at the wall of the channel is ensured by an accurate feeding system controlled by sonic throats. The injection velocity is assumed to be normal to the wall and with a constant norm in space. Among the injecting modules, one measurement module monitors pressure, temperature and velocity inside the chamber.

The similarity with full scale motors is based on the conservation of the Mach number but the Reynolds (Reynolds number is expressed as function of the injected mass flow rate and inner radius) number range is one order of magnitude smaller.

\subsection{Vibration measurement of inhibitors}

A non-intrusive measurement system using a laser interferometer, allowing both displacement and frequency measurements of flexible inhibitors, has been chosen in order to measure the inhibitor response under aeroacoustic excitation and to show how it could influence the system behavior. Laser Velocimeters work on the Laser Doppler principle: the vibration velocity of a point is directly proportional to the Doppler shift.

The VALDO setup with a non reflective head end and with the Laser vibrometer sensor head is displayed in Figure 2. Within the framework of measurement technique validation, experimental preliminary tests, not depicted here, have been 


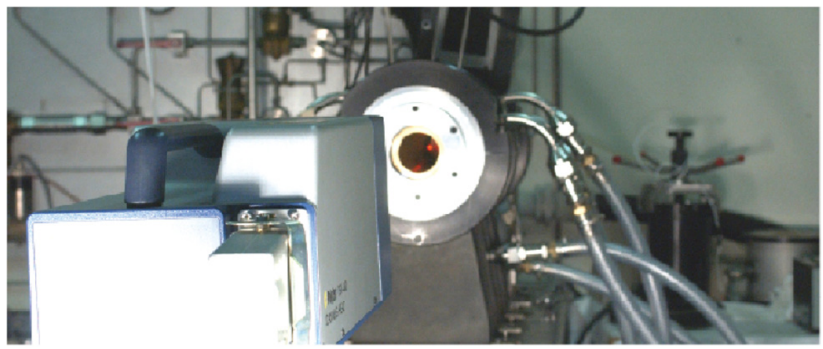

Figure 2: View of the apparatus used for vibration measurement (VALDO setup, laser head with automatic scanning on the inhibitor surface.)

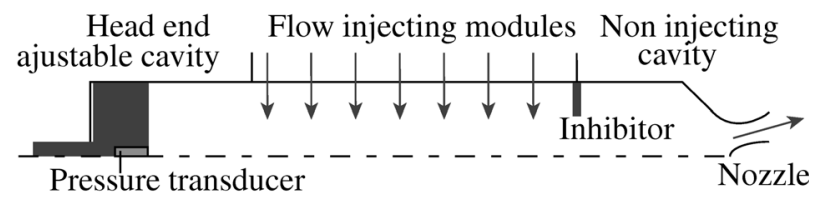

Figure 3: VALDO configuration used for aeroacoustic investigations.

undertaken and demonstrate the ability of this measurement technique to fulfill the need of vibration measurement of inhibitors.

\subsection{Aeroacoustic lock-in}

Vortex shedding and acoustic interaction was performed using rigid, sharp-edged, aluminum discs with very high resonant frequencies relative to the natural acoustic frequencies of the chamber. The experimental setup used for these tests is a simplified geometry (Figure 3). Geometrical values are given in Table 1.

The following variations in the experimental configuration were used to generate flow-induced acoustic pressure responses:

- Natural acoustic frequencies of the test section were varied by adjusting the head end cavity length and calculated as follows:

$$
f_{a c}=\frac{a}{2\left(l_{\text {head }}+l_{\text {inj }}+l_{\text {int }}\right)},
$$

with $l_{\text {head }}$ the length of the Head end cavity and $a$ the speed of sound.

- Injection flow velocity continuously varied at a fixed head end cavity width.

Acoustic pressure responses to vortex shedding were measured, for both variations, with a pressure transducer located at the head end of the test section.

Flow-acoustic coupling involving one inhibitor close to the nozzle was previously studied in a purely axial injected flow [13]. In this study, large pressure fluctuation values happened when vortex shedding from inhibitor was coupled to one of the natural acoustic frequency of the chamber. This lock-in phenomenon 
Table 1: VALDO geometrical parameters for the first experimental case.

\begin{tabular}{|c|c|c|}
\hline Cavity & Inner diameter $(\mathrm{mm})$ & Thickness/width $(\mathrm{mm})$ \\
\hline Head-end $\left(l_{\text {head }}\right)$ & 60 & 0 to 168 \\
\hline Intersegment $\left(l_{\text {int }}\right)$ & 60 & 40 \\
\hline Inhibitor & 45 & 1 \\
\hline Injecting module $\left(l_{\text {inj }}\right)$ & 60 & 168 \\
\hline
\end{tabular}

was investigated by authors over a parametric study varying inhibitor to nozzle distance, $l$, and inhibitor height, $h$. Experimental findings state the ratio $\frac{l}{h}$ as a fundamental parameter with a critical value of 12 under which flow acoustic coupling remains possible. In our experiment configuration, $\frac{l}{h}$ is about 5 .

\subsubsection{Results and discussion}

First experimental test: Tests have been performed with one rigid inhibitor placed in the test section for an operating injection velocity of $0.96 \mathrm{~m} \cdot \mathrm{s}^{-1}$. Then, head end cavity was linearly opened from $0 \mathrm{~mm}$ to $168 \mathrm{~mm}$. Pressure spectrum, Figure 4, is mainly characterized by two dominant, nearly constant, frequencies over the cavity width range. A first peak is observed around $350 \mathrm{~Hz}$ and after an abrupt jump, a starting second one at $300 \mathrm{~Hz}$. It must be remembered that flow injection condition remains the same during the whole test. The maximum of sound pressure level is observed experimentally, Figure 4, when the natural acoustic frequency of the chamber coincides with the frequency of measured pressure fluctuations. Acoustic resonance enhances a high level of pressure oscillations. Therefore, if vortex shedding occurs, when its frequency is close to a natural acoustic frequency of the chamber, then high pressure levels are reached. The frequency jump, for a $150 \mathrm{~mm}$ length head end cavity, indicates that an aeroacoustic phenomenon tends to lock itself on acoustic frequencies.

Second experimental test: The head end cavity had a fixed length of $65 \mathrm{~mm}$ and injection velocity was varied from $0.9 \mathrm{~m} . \mathrm{s}^{-1}$ to $1.7 \mathrm{~m} . \mathrm{s}^{-1}$. Whenever dominant frequency is clearly discernible, a linear evolution with the increase of velocity is observed. Frequency jump (Figure 5) also indicates that vortex shedding is locked on the first acoustic mode. For an injection velocity lower than $1.4 \mathrm{~m} . \mathrm{s}^{-1}$, dominant frequencies lie in the range of about $350-450 \mathrm{~Hz}$. Abrupt changes in dominant frequencies with small changes in velocity are observed for an injection velocity about $1.16 \mathrm{~m} \cdot \mathrm{s}^{-1}$. Again, the maximum sound pressure level is observed when aeroacoustic frequency crosses the natural acoustic frequency of the chamber. Vortex shedding frequency locks itself on acoustic frequencies.

In these experiments, there are no hot wire measurements allowing precise determination of the vortex shedding frequency. As a consequence, it can't be clearly determined which type of vortex shedding is involved in the aeroacoustic process 
154 Fluid Structure Interaction V
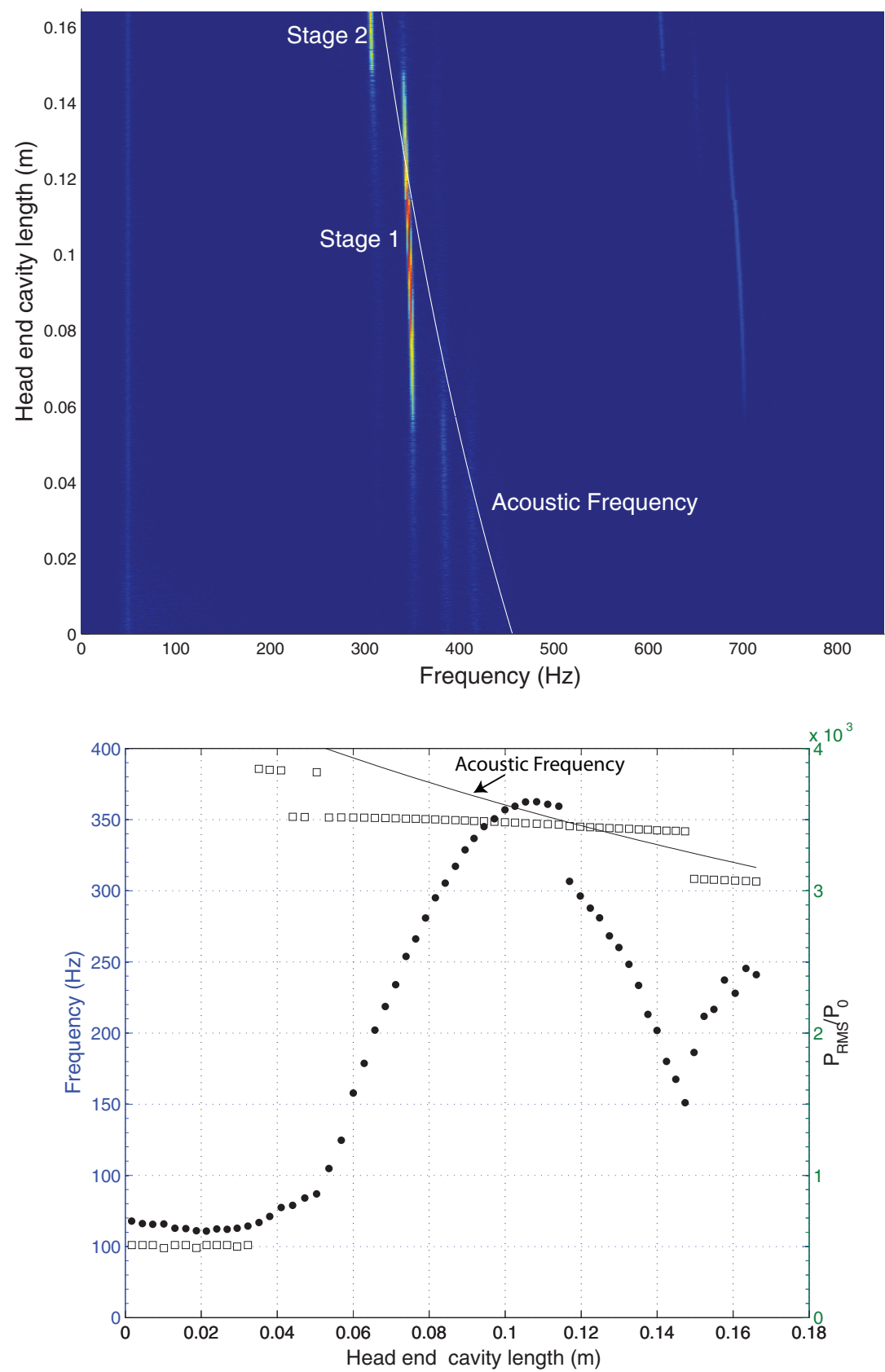

Figure 4: (Top) Frequency spectrum. (Bottom) RMS amplitude of pressure fluctuations as a function of head end cavity length $\left(V_{i n j}=0.96 \mathrm{~m} . \mathrm{s}^{-1}\right)$. Line (-) represents the first acoustic mode of the chamber. 

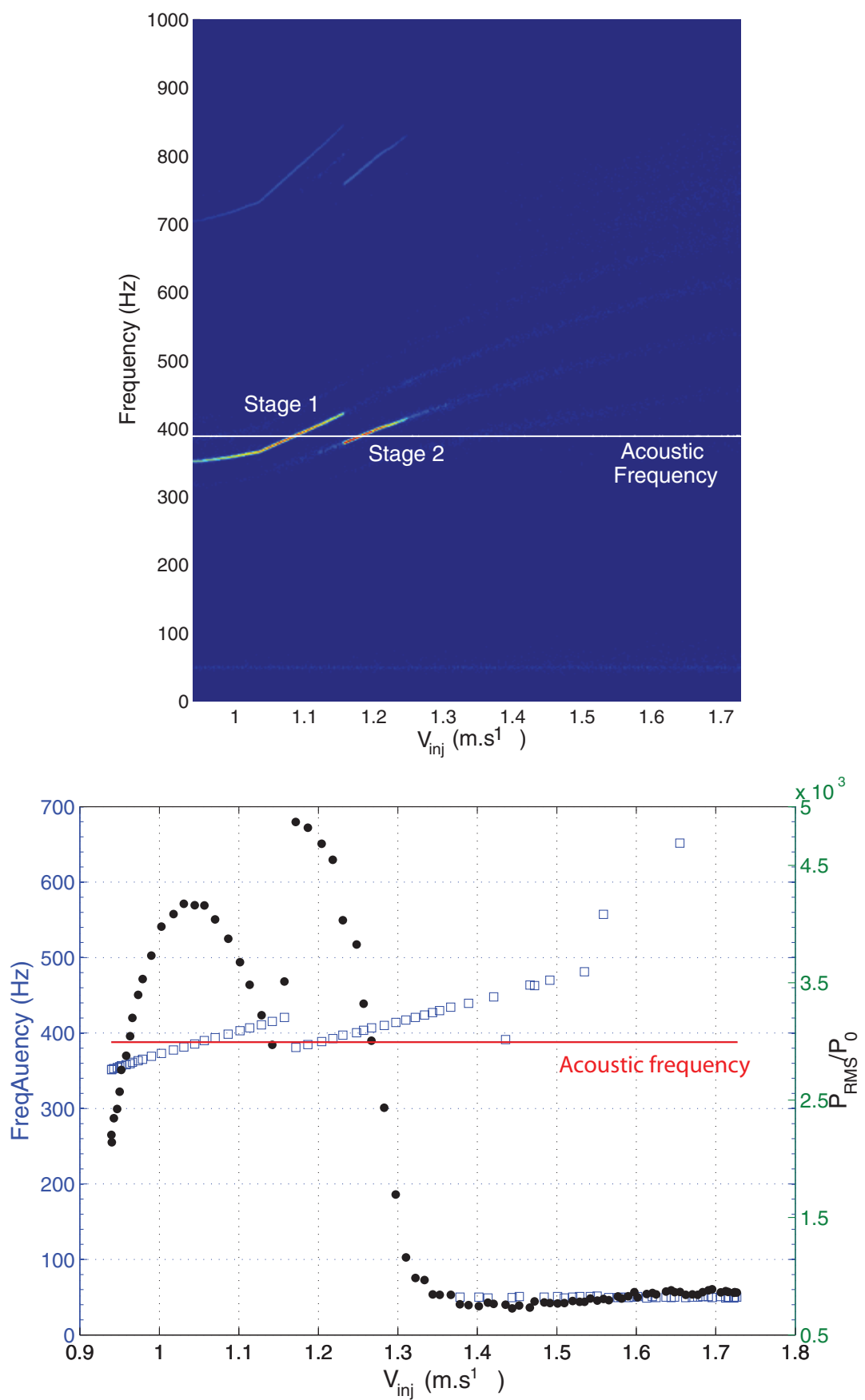

Figure 5: (Top) Frequency spectrum. (Bottom) RMS amplitude of pressure fluctuations as a function of $V_{i n j}$. Line (-) represents the first acoustic mode. 


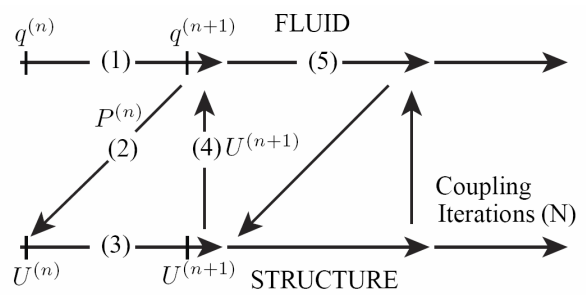

Figure 6: FSI numerical procedure.

(see [12]). Indeed, two potential sources of vortex shedding are present in such a configuration:

- the Taylor Culick flow induced vortex shedding, well studied by Casalis and al. [10],

- and the shear layer instability. Jet tones are produced when unstable jet flow induced by an orifice is amplified by feedback generated as the jet impinges on a downstream surface.

Further analysis will try to discern which kind of instability is meanly involved in pressure oscillations inside VALDO apparatus.

\section{Numerical study of a quasi-static case}

\subsection{Coupling procedure}

In the coupled approach used here, the FSI is achieved by partitioning the problem into fluid and solid parts solved separately with boundary conditions calculated by the other part. As a step to a fully dynamic FSI calculation, a steady state approach is first undertaken.

Inertial properties of the structure are neglected and fluid pressure is applied as a distributed load on the structure The coupling procedure is the same used, few years ago by Roach et al. [15]. Figure 6 shows the sequence of iteration steps. It starts with the calculation of the aerodynamic field until reaching a steady state (path 1). The resulting pressure distribution $P$ is transferred to the finite element nodes (path 2). Using this new interface conditions the structural code computes the deformation $U$ of the structure (path 3). The resulting displacements modify the fluid surface grid and consequently change the boundary conditions (path 4) but also the entire grid in the fluid domain in the next step (path 5).

\subsection{The structural (ZeBuLoN) and flow (CEDRE) solver}

The partitioned FSI procedure developed at ONERA involve the two solvers, CEDRE and ZeBuLoN, for respectively flow and structure subsystems. The CEDRE code is a general polyhedra unstructured computational CFD solver in the field of energetics, with particular emphasis on propulsion applications [19]. 
Its compressible, reactive flow module solves the Navier-Stokes equations with any number of species and various models for chemical reactions. In this paper, the equations are solved using a second-order upwind discretization scheme based on Roe's flux difference splitting. The time integration is obtained by an explicit Runge-Kutta second order method. ZeBuLoN is an advanced object FEA solver with many non-linear solution procedures for material oriented analysis.

\subsection{FSI experimental static reference case}

One of the main objectives of this experimental test is to provide data aiming at validating the coupled computations. The VALDO set-up has been used in a configuration representative of SPRM. The geometry is composed of three injecting modules, a non injecting intersegment and an aft end cavity with submerged nozzle. A $2 \mathrm{~mm}$ thick inhibitor of elastomeric material is clamped between the intersegment cavity and the third injecting module. Parametric details are given in Figure 7.

The non intrusive measurement system presented in Section 3 provides measurement of inhibitor's vibration velocity measurement. Displacements (shown in Figure 8 ) are then computed by integrating the velocity vibration over time. Air is injected at $1.19 \mathrm{~m} . \mathrm{s}^{-1}$ inside the chamber and structure deflection due to fluid stress loading is monitored.

Under fluid stress loading, for an internal pressure chamber of about 2 bar, a disparity in pressure on each side of the inhibitor creates a bending moment. As a consequence, the inhibitor bends and reaches a quasi-steady state with a maximum deflection $d_{\max }$ on its top, about $3 \mathrm{~mm}$ (precisely $d_{\max }=3.285 \mathrm{~mm}$ ). Such experimental data provides values of deflection that can be used for coupled simulation validation.

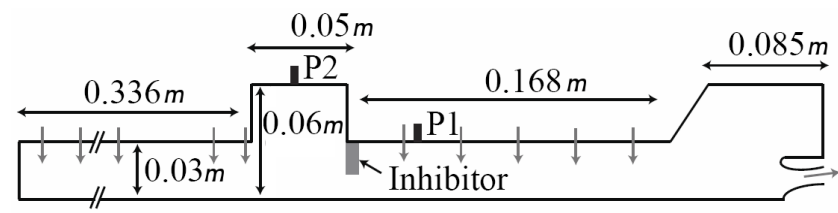

Figure 7: VALDO configuration used for static reference case.
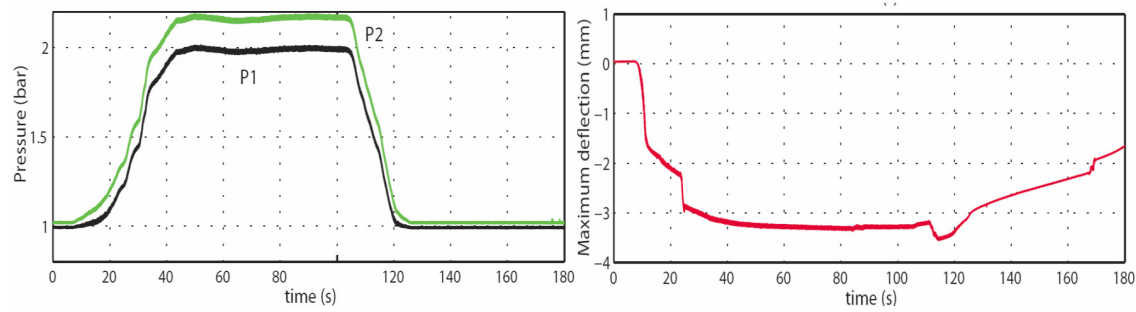

Figure 8: (Left) Pressure evolution. (Right) Maximum deflection of the inhibitor. 

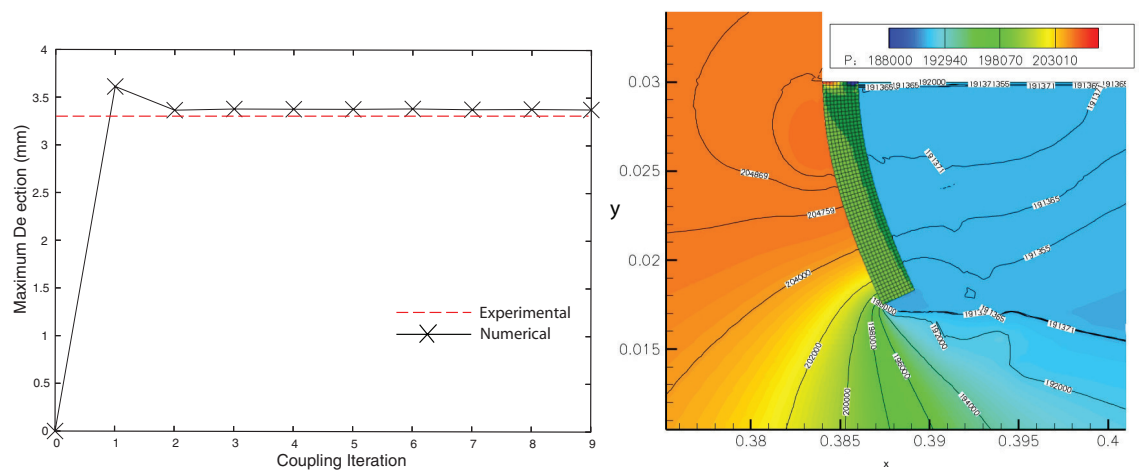

Figure 9: (Left) Maximum deflection of the inhibitor as a function of the number of coupling iterations. (Right) Mean pressure field and deflection of the inhibitor.

\subsection{Numerical model: material and gas properties}

The gas is assumed to be ideal air at $T=20^{\circ} \mathrm{C}$. Inhibitor $\left(\rho=1100 \mathrm{~kg} \cdot \mathrm{m}^{3}\right)$ is assumed to obey non-linear Mooney constitutive model (within a Total Lagrangian framework), with quasi-incompressible assumption and a Young modulus initially set to $13.8 \mathrm{MPa}$. Rheological parameter was set by preliminary mechanical tests not detailed herein. The fluid and structural meshes are respectively constituted of 18685 cells and 2011 nodes and are perfectly matched.

\subsection{Numerical results}

Ten coupling iterations were completed. The maximum deflection of the inhibitor for each coupling iteration is plotted in Figure 9. Quasi-steady deflection seems to be reached after 3 coupling iterations. Good agreement between experiment and simulation is obtained. However, a slight difference indicates that rheological parameters could be optimized.

One can obviously notice (Figure 9) that taking into account the deflection of the inhibitor changes the pressure distribution along the chamber and may have serious impact on pressure fluctuation prediction. FSI inside SPRM is thus a major issue and must be taken into account when computing such configurations.

\section{Conclusion and future work}

Coupling phenomenon between acoustics and hydrodynamics was first experimentally investigated with the VALDO cold flow apparatus. Pressure fluctuations measurements indicate high level when vortex shedding frequencies organize in the 
vicinity of the acoustic mode. Within a certain range of velocity, abrupt frequency shift occurs suggesting a locking-on phenomena between acoustics and hydrodynamics. Nevertheless, vortex shedding may result either from the shear layer instability or from the natural instability of the radially injected flow and therefore further investigation about this issue will be undertaken.

Regarding FSI inside SPRM, a non intrusive measurement technique was applied to the cold flow setup allowing both displacement and frequency measurements of a flexible inhibitor. Preliminary tests have been undertaken on a configuration representative of SPRM and allow determination of deflection of the flexible inhibitor. A numerical coupling procedure, in a quasi-steady approach, was developed and validated on the experimental configuration. This constitutes a first step in the validation process of this coupling method that will be pursued in the future in a fully dynamical way.

\section{References}

[1] Culick, F., Unsteady motions in combustion chambers for propulsion systems. NATO Research and Technology Organisation, 2006.

[2] Scippa, S., Pascal, P. \& Zanier, F., Ariane 5-mps - chamber pressure oscillations full scale firing results: Analysis and further studies. AIAA Paper $94-$ 3068, 1994.

[3] Flandro, G.A. \& Jacobs, H.R., Vortex generated sound in cavities. AIAA Paper 73-1014, 1973.

[4] Flandro, G.A., Vortex driving mechanism in osillatory rocket flows. Journal of Propulsion and Power, 2(3), pp. 206-214, 1986.

[5] Culick, F.E.C. \& Magiawala, K., Excitation of acoustic modes in a chamber by vortex shedding. Journal of Sound and Vibration, 64(3), pp. 455-457, 1979.

[6] Dunlap, R. \& Brown, R.S., Exploratory experiments on acoustic oscillations driven by periodic vortex shedding. AIAA Journal, 19(3), pp. 408-409, 1981.

[7] Brown, R., Dunlap, R., Young, S. \& Waugh, R., Vortex shedding as an additional source of acosutic energy in segmented solid propellant rocket motor. Journal of Spacecraft and Rocket, 18, pp. 312-319, 1981.

[8] Nomoto, H. \& Culick, F., An experimental investigation of pure tone generation by vortex shedding in a duct. Journal of Sound and Vibration, 84(2), p. $247252,1982$.

[9] Vuillot, F., Vortex-shedding phenomena in solid rocket motors. Journal of Propulsion and Power, 11, p. 626639, 1995.

[10] Chedevergne, F., Casalis, G. \& Feraille, T., Biglobal linear stability analysis of the flow induced by wall injection. Physics of Fluids, 18(1), 2006.

[11] Lupoglazoff, N. \& Vuillot, F., Parietal vortex shedding as a cause of instability for long solid propellant motors. 34th AIAA Aerospace Sciences Meeting, AIAA Paper 96-0761, January 1996. 
[12] Vetel, J., Plourde, F. \& Doan-Kim, S., Characterisation of a coupled phenomenon in a confined shear-layer. International Journal of Heat and Fluid Flow, 23(4), pp. 533-543, 2002.

[13] Anthoine, J. \& Olivari, D., Investigation of flow acoustic coupling in a model of solid propellant boosters. Aerothermodynamics for space vehicles, ed. R.A. Harris, volume 426 of ESA Special Publication, p. 583, 1999.

[14] Avalon, G., Ugurtas, B., Grisch, F. \& Bresson, A., Numerical computations and visualization tests of the flow inside a cold gas simulation with characterization of a parietal vortex shedding. 36th AIAA/SAE/ASME Joint Propulsion Conference and Exhibit, AIAA Paper 2000-3387, July 2000.

[15] Roach, R., Gramoll, K., Weaver, M. \& Flandro, G., (eds.), Fluid-structure interaction of solid rocket motor inhibitors, 1992.

[16] Wasistho, B., Fiedler, R., Namazifard, A. \& Brandyberry, M., 3-d coupled simulations of flexible inhibitors in the rsrm. AIAA Paper 2005-3996, AIAA, SAE, ASME, and ASEE, 41st Joint Propulsion Conference and Exhibit, Tucson, Arizona, 2005.

[17] Rey, C., Froment, V., Errera, M.P., Truffart, B. \& Langlois, A., Numerical investigation of fluid-structure interaction in a model of solid propellant motors. 2nd European Conference for Aerospace Sciences (EUCASS), 2007.

[18] Avalon, G. \& Josset, T., Cold gas experiments applied to the understanding of aeroacoustic phenomena inside solid propellant boosters. 42nd AIAA/ASME/SAE/ASEE Joint Propulsion Conference and Exhibit, AIAA Paper 2006-5111, July 2006.

[19] Bertier, N., Courbet, B., Dutoya, D., Vuillot, F. \& Sagaut, P., Large-eddy simulation of a subsonic flow over a cavity on general unstructured grids. AIAA 2004-0679, 42nd AIAA Aerospace Sciences Meeting and Exhibit, Reno, January 5-8, 2004. 